\title{
Biologicals and how they revolutionized rheumatology
}

\author{
Johannes Grisar
}

Published online: 21 January 2015

(C) Springer-Verlag Wien 2015

About one and a half decades ago, biological therapies have started to challenge our therapeutic options in rheumatology. Until the late 1990s, the rheumatologist's armamentarium consisted mainly of methotrexate and other disease-modifying drugs (DMARDs) like sulfasalazine, D-penicillamine, gold and cyclosporine as well as medium to high doses of glucocorticoids and non-steroidal anti-rheumatic drugs (NSAIDs). Although many patients could be treated sufficiently, a noteworthy proportion of them still suffered from rapidly progressing disease despite receiving conventional immunosuppressive drugs. Moreover, these patients often developed several side effects due to the high-dose glucocorticoid and NSAID intake associated with increased morbidity and mortality.

However, this scenario began to change when the first biological drugs, or the so-called targeted therapies, were approved around the millennium. During the 1980s, our pathophysiologic understanding of inflammatory rheumatic diseases and the immune processes involved in triggering them increased rapidly [1]. The cytokine tumour necrosis factor (TNF) was found to be pivotally involved in the perpetuation of the disease process leading to the development of the first mononuclear antibody named infliximab that directly targeted TNF and was initially only approved for rheumatoid arthritis (RA). This mouse-human chimeric antibody showed a significant improvement when compared with the conventional DMARDs. After this "ignition," in the following years, several other TNF-targeting antibodies were approved. They all exhibited slight differences regarding

J. Grisar, MD $(\bowtie)$

2nd Department of Internal Medicine with

Rheumatology/Osteology and Gastroenterology,

KH Barmherzige Schwestern (St. Vincent Hospital),

Stumpergasse 13, 1060 Vienna, Austria

e-mail: johannes.grisar@bhs.at their molecular structure and were applied subcutaneously instead of intravenously.

Besides their highly convincing data in RA, TNF blockade also was shown to be highly effective in axial spondyloarthritis and psoriatic arthritis as demonstrated by several articles in this special issue (Kocijan et al.). Especially in ankylosing spondylitis and non-radiographic axial spondyloarthritis that were mainly treated with NSAIDs so far and which were only effective in slightly more than a half of the patients, a new era began.

In RA however, several other molecules and pathways, like interleukin-6, co-stimulation and CD20 that are involved in the disease process, could be targeted effectively and further expanded our possibilities to treat this crippling disease in a sufficient manner (Blüml et al., Rath et al., Scheinecker et al.). The fact that targeting these molecules failed in the other diseases where anti-TNF was effective underlines the differences in the pathways of these inflammatory diseases. In contrast, blocking the IL-17/IL-23 pathway was shown to be beneficial in psoriatic arthritis (Kocijan et al.).

The development of biological therapies, however, pushed rheumatology from a 'low-cost' discipline to the other extreme. Nowadays, some of the biological therapies are amongst the top with regards to earnings and revenue of the pharmaceutical industry.

In the next years, the majority of patents of biological therapies will expire, opening the market for the so-called biosimilars that are produced from other than the originator companies. Due to the complex structure and the manufacturing process of the biologicals, there is an ongoing discussion how similar biosimilar drugs really are and whether there might be differences with regards to efficacy and safety on the long term when compared with the originator drugs. The expected price reduction of biologicals, however, may make these drugs available to a broader population of patients with chronic inflammatory diseases in countries that hardly had access to these drugs so far. 


\section{editorial}

In this issue, the biological therapies in rheumatology, their mode of action and and their distinct indications will be unravelled by experts in the field of the distinct inflammatory and rheumatic diseases.

\section{Conflict of interest}

The author declares no conflict of interest.

\section{Reference}

1. Smolen J, Steiner G. Therapeutic strategies for rheumatoid arthritis. Nat Rev Drug Discov. 2003;2:473-88. 